\title{
CEREBRAL RSO2 MONITORING DURING SHUNT TAP IN MALFUNCTIONING VENTRICULAR SHUNT
}

\author{
T. Abramo ${ }^{1}$, C. Estrada ${ }^{1}$, C. Moore ${ }^{1}$, D. Arnold ${ }^{1}$, C. Zhou ${ }^{2}$
}

${ }^{I}$ Department of Pediatrics, Division of Pediatric Emergency Medicine, Vanderbilt Medical University, Nashville, TN, ${ }^{2}$ Pediatrics/Adjunct, Health Services, Seattle Children's Research Institute, University of Washington, Seattle, WA, USA

Background and aim: Shunt tap decreases ICP \& improves cerebral perfusion \& should be detected by cerebral rSO2.Determine cerebral rSO2 reliability during shunt tap.

Method: Left \& right cerebral rSO2 q 5 seconds before,during \&1 hour posttap.

Results: 94 subjects had cerebral rSO2 monitoring, 63 proximal \& 31 distal malfunctioning.

Overall post-pretap $\mathrm{rSO}$, right hemisphere showed significant difference, $\mathrm{p}=0.03$. Post-pretap linear regression mean,Left:-0.17,95\%CL:-0.27,-0.06,p=0.002, Right:-0.12,95\%Cl:-0.22,-0.02,p=0.019) \& SDLeft:-0.68, 95\%CI:-0.9,-0.5,p< 0.001,Right:-0.64 95\%CI:-0.82,-0.45,p<0.001 were significant. 94 pts' Bland-Altman test showed wide between cerebral difference $-0.28,95 \% \mathrm{CI}$ :-18.1,17.5. Distal vs proximal preposttap linear regression was predictive for site. (Left:-0.48,95\%CI:-0.72,-0.24,p< 0.001, Right:$0.23,95 \% \mathrm{CI}:-0.43,-0.018, \mathrm{p}=0.033)$.

\begin{tabular}{|c|c|c|c|c|c|c|c|c|}
\hline $\begin{array}{l}\text { Probe Position } \\
\text { Forehead } \\
\text { Ages:mean4.8yrs } \\
\text { SD4.4 } \pm\end{array}$ & Status & Mean & S.D. & Median & & & & \\
\hline $\begin{array}{l}\text { Left Forehead } \\
\text { rSO2 }\end{array}$ & Pre-tap & 68.9 & 11.0 & 68.5 & & & & \\
\hline $\begin{array}{l}\text { Left Forehead } \\
\text { rSO2 }\end{array}$ & During Tap & 69.1 & 11.1 & 69.7 & & & & \\
\hline $\begin{array}{l}\text { Left Forehead } \\
\text { rSO2 }\end{array}$ & Post-Tap & 69.6 & 10.3 & 71.9 & & & & \\
\hline $\begin{array}{l}\text { Right Forehead } \\
\text { rSO2 }\end{array}$ & Pre-Tap & 69.7 & 9.2 & 70.9 & & & & \\
\hline $\begin{array}{l}\text { Right Forehead } \\
\text { rSO2 }\end{array}$ & During-Tap & 70.7 & 9.0 & 70.4 & & & & \\
\hline $\begin{array}{l}\text { Right Forehead } \\
\text { rSO2 }\end{array}$ & Post-Tap & 70.7 & 8.9 & 71.4 & & & & \\
\hline $\begin{array}{l}\text { ICC Analysis for } \\
\text { Pre \& Post Tap }\end{array}$ & N 94 & $\begin{array}{l}\text { Left Forehead } \\
\text { ICC }\end{array}$ & $95 \%$ LL & $95 \% \mathrm{UL}$ & $\mathrm{N}$ & $\begin{array}{l}\text { Right Forehead } \\
\text { ICC }\end{array}$ & $95 \% \mathrm{LL}$ & $95 \%$ UL \\
\hline Pre-Tap & 60095 & 0.86 & 0.82 & 0.89 & 60095 & 0.80 & 0.76 & 0.85 \\
\hline Post-Tap & 40761 & 0.79 & 0.74 & 0.84 & 40761 & 0.81 & 0.77 & 0.85 \\
\hline
\end{tabular}

[Cerebral rSO2 94 Subjects]

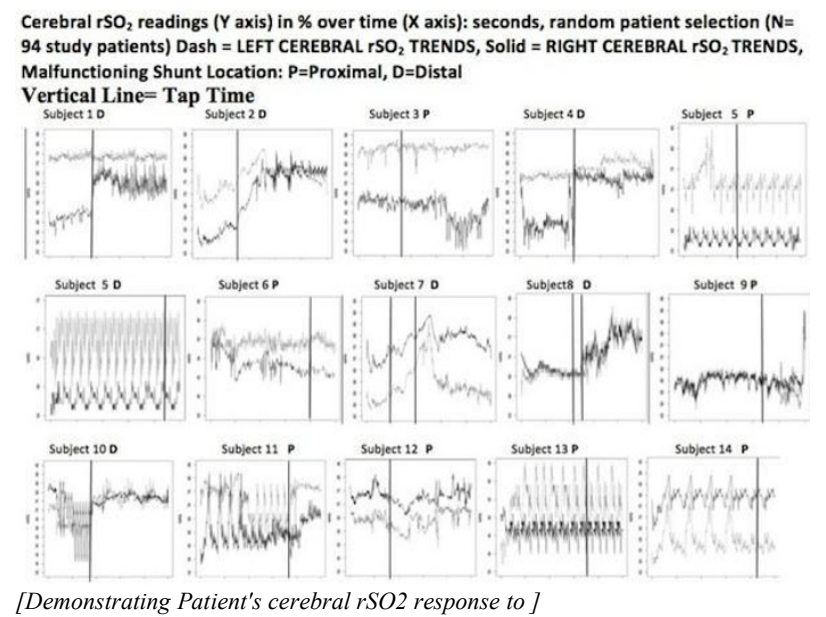

Conclusion: Cerebral rSO2 monitoring during shunt taps demonstrated cerebral perfusion changes with CSF removal. rSO2 readings were significantly different after tap; more in distal vs proximal malfunction. 